\title{
Evaluation of pregnancy-associated plasma protein-A and some inflammation markers for atherosclerosis in psoriasis patients
}

\author{
Fikret Akyurek ${ }^{1}$, Fatma Tuncez Akyurek ${ }^{2}$ \\ 'Department of Biochemistry, Faculty of Medicine, Selcuk University, Konya, Turkey \\ 2Department of Dermatology, Faculty of Medicine, Selcuk University, Konya, Turkey
}

Adv Dermatol Allergol 2021; XXXVIII (1): 151-155

DOI: https://doi.org/10.5114/ada.2021.104290

\begin{abstract}
Introduction: Psoriasis is a common, chronic inflammatory disease of the skin. Although its relationship with cardiovascular risk factors is one of the main causes of mortality, the association between psoriasis and cardiovascular events is controversial.

Aim: The aim of this study is to measure the levels of hs-CRP and pregnancy-associated plasma protein-A (PAPP-A) related to atherosclerosis in patients with moderate-severe psoriasis and to evaluate their possible association with disease severity.

Material and methods: This study included 45 moderate-severe psoriasis patients without additional risk factors for cardiovascular disease and 45 healthy individuals as a control group.

Results: Serum PAPP-A levels in both groups were given as two different variables, detectable and non- detectable, based on a value of $0.004 \mathrm{U} / \mathrm{LL}$. When PAPP-A levels were compared, there was no statistically significant difference between the groups.

Conclusions: The use of the PAPP-A test in the evaluation of early-stage atherosclerosis in moderate-severe psoriasis patients who do not have additional risk factors for cardiovascular diseases may not be sufficient.
\end{abstract}

Key words: psoriasis, pregnancy-associated plasma protein-A, atherosclerosis, high-sensitivity C-reactive protein.

\section{Introduction}

Psoriasis is a common, chronic inflammatory disease of the skin [1]. It affects $2-5 \%$ of people around the world. Psoriasis is defined as the excessive increase in keratinocytes or epidermal hyperplasia [2]. In live cells, the cell cycle is regulated by various proteins. Deviations in the mechanisms that regulate the cell cycle may lead to abnormal proliferation in the respective cells. These proteins, which take part in the regulation of the cell cycle, play a key role in maintaining typical and abnormal cell growth and cell proliferation [2]. It has been shown that mitosis in these cells is 50 times greater than in normal cells. Cells in mitosis have been shown to reproduce 50 times faster than normal epidermis [3].

In recent years, psoriasis has been associated with various comorbidity. It is also considered a systemic inflammatory disease today. The disease has been described as a link between obesity, dyslipidaemia, type 2 diabetes mellitus (DM) and hypertension (HT). This relationship may be due to genetic, environmental and immunological factors. Factors such as proinflammatory cytokines and increased oxidative stress may support leukocyte adhesion and thrombotic processes [1]. Systemic inflammation may occur in $7-26 \%$ of psoriatic patients. Inflammation can be assessed by analysis of blood cell count and other biochemical markers. The neutrophil count, lymphocyte count and neutrophil-lymphocyte ratio (NLR) are valuable indicators of subclinical systemic inflammation [4]. In psoriasis patients all these pathologies may cause higher cardiovascular disease morbidity and mortality [5]. However, not all studies support a link between psoriasis and cardiovascular risk factors. This issue is still controversial.

Pregnancy-associated plasma protein-A (PAPP-A) plays a key role in development and aging processes by

Address for correspondence: Fikret Akyurek MD, Department of Biochemistry, Faculty of Medicine, Selcuk University, Konya, Turkey, phone: +90 5053947980, e-mail: dr.akyurek@hotmail.com Received: 1.04.2019, accepted: 28.05.2019. 
modulating the effects of growth hormone $(\mathrm{GH})$ on the cardiovascular system, as well as on glucose, protein and lipid metabolism [6]. Both excess and deficiency of GH are associated with cardiovascular diseases. In the experimental models, GH and its main mediator, insulin-like growth factor-1 (IGF-1), have been shown to have both proatherogenic and antiatherogenic effects [7]. GH levels are associated with body fat, protein and carbohydrate ratios, and PAPP-A is released more than fat in visceral regions compared to subcutaneous fats. Conversely, PAPP-A decreases in mesenteric fat depots of knock-out mice. This indicates an association between adipose tissue and PAPP-A. There is a negative relationship between PAPP-A and body mass index (BMI) [8]. High plasma PAPP-A concentration is associated with the degree of coronary artery disease. High blood levels of PAPP-A indicate an increased risk of death. PAPP-A can be considered as a prognostic marker for chronic stable angina pectoris and acute coronary syndromes. A positive association between PAPP-A levels and carotid and peripheral arterial disease has been reported [8]. A relationship was found between PAPP-A and anti-inflammatory effective cytokine interleukin-10 (IL-10), which are indicating that PAPP-A is only harmful when IL-10 levels are low [8, 9].

Inflammatory reactions in various diseases are considered to be mediated by JAK/STAT intracellular signalling pathways [10-14]. A transcription factor protein regulated by JAK/STAT contributes to various cellular activities with the regulation of various cytokine signalling pathways [15]. The activated STAT has an important role in the functioning of genes that govern various biological processes such as cell growth, cell division, apoptosis, and immune reactions [16].

To confirm the diagnosis, although histological evaluation of skin biopsies in the psoriasis patients is a widelyused method, the need for new scientific methods has increased due to the limitations of the procedure such as invasiveness and inability to diagnose before lesion formation. Therefore, the determination of changes in biomarkers which decrease or increase in the course of the disease may be an important step in terms of the etiopathology, predictability and treatment of psoriasis [2].

\section{Aim}

The aim of this study is to measure the levels of PAPP-A, hs-CRP and white blood cells associated with atherosclerosis in patients with moderate-severe psoriasis and to evaluate their possible association with disease severity.

\section{Material and methods Study design}

In this study, 45 psoriasis patients who attended the dermatology clinic of the Selcuk University, Faculty of
Medicine and did not have any additional risk factors for cardiovascular diseases were included. As a healthy control group, 45 healthy individuals who were admitted to our hospital for general health screening and who could not detect any disease were included in the study. The patient and control groups were composed of 22 females and 23 males. The ages of the patient and control groups were similar. Patients with a psoriasis area and severity index (PASI) greater than 10 were included in the study. Patients with any additional diseases other than psoriasis were excluded from the study.

For routine examinations, blood was collected from the antecubital region of patient and control groups by using a vacutainer in the sitting position after fasting for $12 \mathrm{~h}$. Serum samples were centrifuged 25 min after being collected. They were centrifuged at $3000 \mathrm{rpm}$ for 10 min and the serums were separated. Routine biochemical tests were performed from serum samples. The remaining serums from routine examinations were separated into Eppendorf tubes and stored at $-80^{\circ} \mathrm{C}$ under appropriate conditions. Complete blood count was performed on Beckman Coulter LH 780 devices as soon as the blood reached the laboratory. Leukocyte (WBC), neutrophil, and lymphocyte counts were reported as $\times 10^{3}$. hs-CRP was analysed by the immunoturbidimetric method in Beckman Coulter AU5800 Series (Japan) device and the unit was mg/dl. PAPP-A tests were analysed with Roche Cobas 601 (Japan) by using the electrochemiluminescence method. The unit of PAPP-A was $\mathrm{U} / \mathrm{I}$. The quantitation limit for PAPP-A is $0.004 \mathrm{U} / \mathrm{I}$. The study was approved by the Local Ethics Committee of the Selcuk University, Faculty of Medicine (approval number 2019/58).

\section{Statistical analysis}

Statistical analysis was performed with SPSS Statistics 21.0. A normal distribution evaluation was applied. Analyses showing non-parametric distribution were evaluated with the Mann-Whitney $U$ test. Categorical variability was expressed in $\%$ and evaluated by $\chi^{2}$ test. The level of statistical significance was $p<0.05$.

\section{Results}

Forty-five patients with psoriasis and 45 healthy individuals were included in the study. Patient and control groups were similar in terms of gender and age. Individuals in both groups had no history of metabolic disease, cardiovascular disease, smoking and alcohol use. The rate of PAPP-A $<0.004 \mathrm{U} / \mathrm{I}$ was $22.2 \%$ in the patient group and $33.3 \%$ in the control group. In the patient group, the rate of $>0.004 \mathrm{U} / \mathrm{I}$ was $66.6 \%$ and it was $77.7 \%$ in the control group. No statistically significant difference was found between those lower than 0.004 and those higher than 0.004 of the patient and control groups ( $p=0.347$ ). Percentages for PAPP-A were evalu- 
ated by the $\chi^{2}$ test. The comparative results of PAPP-A according to the quantitation limits are shown in Figure 1 and Table 1. Since PAPP-A (> 0.004), hs-CRP, neutrophil, lymphocyte and neutrophil/lymphocyte parameters did not show a parametrical distribution, Mann-Whitney $U$ test was used to compare patient and control groups. As shown in Table 2, data were taken as median (min.max.). Leukocyte counts were found significantly higher in patients than in the control group $(p<0.001)$. Lymphocyte counts were also found significantly higher in the patient group than in the control group ( $p=0.02)$. There was no significant difference in terms of other parameters.

\section{Discussion}

In our study, we investigated the relationship between psoriasis disease and atherosclerosis by comparing PAPP-A and hs-CRP levels of psoriasis patients without a known metabolic disease and a healthy control group.

Atherosclerosis is an important health problem for both developed and developing countries. Acute coronary syndrome (ACS) accounts for $20 \%$ of all medical emergencies and has the highest risk of death. In addition to known risk factors, it is reported that some new risk factors are associated with ACS. Because of the inability of routine tests to give clear information about the atherosclerotic process, new biomarkers are needed to evaluate atherosclerosis [17]. PAPP-A and hs-CRP are emerging biomarkers to evaluate plaque stability [18]. PAPP-A is a molecule of glycoprotein structure which is produced by placental syncytiotrophoblast cells and found in serum. However, today it is known that PAPP-A is produced in tissues other than placenta. PAPP-A, a member of the metalloproteinase family, contributes to the deterioration of plaque by disrupting the stability of atherosclerotic plaques [19].

The hs-CRP is a commonly studied marker for atherosclerotic plaques. It is an acute phase reactant and an important agent that shows the progression of atherosclerosis [17]. Our study showed that there was no significant difference between patient and control groups

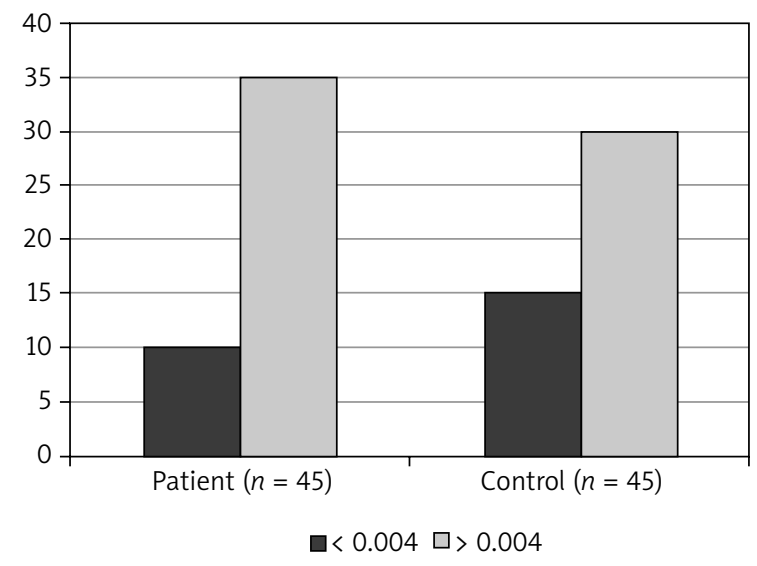

Figure 1. PAPP-A levels in the patient and control groups; detectable $(>0.004 \mathrm{U} / \mathrm{l})$ and undetectable $(<0.004 \mathrm{U} / \mathrm{l})$

for hs-CRP and PAPP-A levels, neutrophil count and NLRs. However, leukocyte and lymphocyte counts were found higher in patients. For PAPP-A, those above and below the measurement limits were grouped and patient and control groups were compared.

In addition, measurable PAPP-A values were compared between groups, but no significant difference was found. PAPP-A is a new biomarker demonstrating atherosclerotic plaque instability. Bayes-Genis et al. were the first to recommend PAPP-A as a biomarker for unstable plaques. They showed that there was a significant relationship between PAPP-A levels and CRP levels in patients with the acute coronary syndrome [20]. It is reported that metabolic and cardiovascular diseases are more common in psoriasis patients [21]. Li et al. found that both levels of hs-CRP and PAPP-A were significantly higher in patients with type 2 diabetes [22]. O'Donoghue

Table 1. Comparison of the serum PAPP-A level in study groups

\begin{tabular}{lccc}
\hline Group & PAPP-A & PAPP-A & \multirow{2}{*}{ P-value } \\
\cline { 2 - 3 } & $<0.004 \mathrm{U} / \mathrm{l}$ & $>\mathbf{0 . 0 0 4} \mathrm{U} / \mathrm{l}$ & \\
\hline Patient $(n=45)$ & $10(22.2 \%)$ & $35(77.7 \%)$ & \multirow{2}{*}{0.347} \\
\cline { 1 - 3 } Control $(n=45)$ & $15(33.3 \%)$ & $30(66.6 \%)$ & \\
\hline
\end{tabular}

Table 2. Laboratory findings in patients with psoriasis and control subjects

\begin{tabular}{|c|c|c|c|}
\hline Parameter & $\begin{array}{c}\text { Patient }(n=30) \\
\text { Median (min.-max.) }\end{array}$ & $\begin{array}{c}\text { Control }(n=35) \\
\text { Median (min.-max.) }\end{array}$ & $P$-value \\
\hline PAPP-A [U/I] & $0.005(0.004-0.013)$ & $0.005(0.004-0.017)$ & 0.26 \\
\hline hs-CRP [mg/l] & $1.61(0.07-26.32)$ & $1.07(0.06-15.88)$ & 0.13 \\
\hline WBC $\left[\times 10^{3}\right]$ & $7.95(3.6-15.4)$ & $6.8(4.8-10.8)$ & $<0.001$ \\
\hline Neutrophil $\left[\times 10^{3}\right]$ & $4.49(1.98-12.16)$ & $3.7(2.11-9.11)$ & 0.05 \\
\hline Lymphocyte $\left[\times 10^{3}\right]$ & $2.45(1.2-3.89)$ & $2.1(0.4-3.3)$ & 0.02 \\
\hline NLR & $1.7(0.7-5.8)$ & $1.8(1-6.1)$ & 0.48 \\
\hline
\end{tabular}


et al. found a significant correlation between hs-CRP and PAPP-A in patients with acute myocardial infarction [23]. Polat et al. found that PAPP-A levels were high in Psoriasis patients, and they also found that PAPP-A levels correlated with the duration and severity of the disease. They suggested that high PAPP-A levels were related with atherosclerosis, and the use of PAPP-A as a screening test for cardiovascular risk assessment should be supported by further studies [24]. Unlike that study, we found no significant difference in moderate-severe psoriasis patients compared to the control group in terms of PAPPA levels as hs-CRP levels, which is an important marker for atherosclerosis. Therefore, we think that the use of PAPP-A test alone will not be sufficient in the evaluation of early-stage atherosclerosis. In a study conducted by Beaudeux et al., there was a significant difference between hyperlipidemic individuals and the control group in terms of PAPP-A levels. The PAPP-A level was found to be low in the control group [25]. In our study, similar results in the patient and control groups may be due to absence of additional systemic diseases such as hyperglycaemia, hyperlipidaemia, obesity and HT in psoriasis patients. These findings made us think that the side effects of psoriasis on the cardiovascular system are limited without additional cardiovascular risk factors. Metabolic diseases and cardiovascular diseases are more common in psoriasis patients. It is controversial whether the increasing frequency of clinical pathologies in psoriasis is associated with psoriasis or whether the disease itself is triggered by these pathologies. In the study of Solak et al.evaluating the relationship between inflammation and psoriasis in 199 patients with psoriasis and 54 healthy individuals, the leukocyte and neutrophil counts and NLR were found to be higher in the patients [26]. In our study, while the leukocyte and neutrophil counts were high in the patients, NLR was lower. The low NLR in patients is due to the high lymphocyte count. Leukocyte counts can change in a short time and often reflect acute conditions. We think that it is not a reliable marker in the diagnosis of chronic conditions such as atherosclerosis.

Lack of radiological evaluation of atherosclerotic plaques, high PAPP-A measurement limits and a limited number of patients can be listed among the limitations of the study.

\section{Conclusions}

In this study, we found no significant difference between the control group and the hs-CRP and PAPP-A levels in patients with moderate to severe psoriasis without any additional disease. Therefore, we think that psoriasis alone does not pose a significant risk of cardiovascular disease development without cardiovascular risk factors such as DM, HT, dyslipidaemia and obesity. Studies with detailed cardiovascular examinations including larger patient series are needed to elucidate this issue.

\section{Conflict of interest}

The authors declare no conflict of interest.

\section{References}

1. Fernández-Armenteros JM, Gómez-Arbonés X, Buti-Soler M, et al. Psoriasis, metabolic syndrome and cardiovascular risk factors. A population-based study. J Eur Acad Dermatol Venereol 2019; 33: 128-35.

2. Yadav K, Singh D, Singh MR. Protein biomarker for psoriasis: a systematic review on their role in the pathomechanism, diagnosis, potential targets and treatment of psoriasis. Int J Biol Macromol 2018; 118: 1796-810.

3. Weinstein GD, van Scott EJ. Autoradiographic analysis of turnover times of normal and psoriatic epidermis. J Invest Dermatol 1965; 45: 257-62.

4. Asahina A, Kubo N, Umezawa Y, et al. Neutrophil-lymphocyte ratio, platelet-lymphocyte ratio and mean platelet volume in Japanese patients with psoriasis and psoriatic arthritis: response to therapy with biologics. J Dermatol 2017; 44: 1112-21.

5. Ogdie A, Troxel AB, Mehta NN, Gelfand JM. Psoriasis and cardiovascular risk: strength in numbers part 3. J Invest Dermatol 2015; 135: 2148-50.

6. Palmeiro CR, Anand R, Dardi IK, et al. Growth hormone and the cardiovascular system. Cardiol Rev 2012; 20: 197-207.

7. Higashi Y, Sukhanov S, Anwar A, et al. Aging, atherosclerosis, and IGF-1. J Gerontol A Biol Sci Med Sci 2012; 67A: 626-39.

8. Nilsson E, Cao Y, Lindholm B, et al. Pregnancy-associated plasma protein-A predicts survival in end-stage renal disease-confounding and modifying effects of cardiovascular disease, body composition and inflammation. Nephrol Dial Transpl 2017; 32: 1776.

9. Mueller T, Dieplinger B, Poelz W, et al. Increased pregnancy-associated plasma protein-A as a marker for peripheral atherosclerosis: results from the Linz peripheral arterial disease study. Clin Chem 2006; 52: 1096-103.

10. Punwani N, Scherle P, Flores R, et al. Preliminary clinical activity of a topical JAK1/2 inhibitor in the treatment of psoriasis. J Am Acad Dermatol 2012; 67: 658-64.

11. Ludbrook VJ, Hicks KJ, Hanrott KE, et al. Griffiths, Investigation of selective JAK1 inhibitor GSK2586184 for the treatment of psoriasis in a randomized placebo-controlled phase Ila study. Br J Dermatol 2016; 174: 985-95.

12. Fridman JS, Scherle PA, Collins R, et al. Preclinical evaluation of local JAK1 and JAK2 inhibition in cutaneous inflammation. J Invest Dermatol 2011; 131: 1838-44.

13. O'Shea JJ, Park H, Pesu M, et al. New strategies for immunosuppression: interfering with cytokines by targeting the Jak/Stat pathway. Curr Opin Rheumatol 2005; 17: 305-11.

14. Chang BY, Zhao F, He X, et al. JAK3 inhibition significantly attenuates psoriasiform skin inflammation in CD18 mutant PL/J mice. J Immunol 2009; 183: 2183-92.

15. Zhuang S. Regulation of STAT signaling by acetylation. Cell Signal 2013; 25: 1924-31.

16. Sehgal PB. Paradigm shifts in the cell biology of STAT signalling. Semin Cell Dev Biol 2008; 19: 329-40.

17. Lodh M, Goswami B, Parida A, et al. Assessment of serum leptin, pregnancy-associated plasma protein A and CRP 
levels as indicators of plaque vulnerability in patients with acute coronary syndrome. Cardiovasc J Afr 2012; 23: 330-5.

18. Blake GJ, Ridker PM. C-reactive protein and other inflammatory risk markers in acute coronary syndromes. J Am Coll Cardiol 2003; 41 (4 Suppl S): 37S-42S.

19. Iversen KK, Dalsgaard M, Teisner AS, et al. Pregnancy-associated plasma protein-a, marker for outcome in patients suspected for acute coronary syndrome. Clin Biochem 2010; 43: 851-7.

20. Bayes-Genis A, Conover CA, Overgaard MT, et al. Pregnancyassociated plasma protein-A as a marker of acute coronary syndromes. N Engl J Med 2001; 345: 1022-9.

21. Pirro M, Stingeni L, Vaudo G, et al. Systemic inflammation and imbalance between endothelial injury and repair in patients with psoriasis are associated with preclinical atherosclerosis. Eur J Prev Cardiol 2015; 22: 1027-35.

22. Li WP, Neradilek MB, Gu FS, et al. Pregnancy-associated plasma protein-A is a stronger predictor for adverse cardiovascular outcomes after acute coronary syndrome in type-2 diabetes mellitus. Cardiovasc Diabetol 2017; 16: 45.

23. O'Donoghue ML, Morrow DA, Cannon CP, et al. Multimarker risk stratification in patients with acute myocardial infarction. J Am Heart Assoc 2016; 5: e002586.

24. Polat M, Bugdayci G, Sahin A, et al. Can pregnancy-associated plasma protein-A be a marker for the assessment of atherosclerosis risk in patients with chronic plaque psoriasis? Adv Dermatol Allergol 2016; 33: 340-4.

25. Beaudeux JL, Burc L, Imbert-Bismut F, et al. Serum plasma pregnancy-associated protein A: a potential marker of echogenic carotid atherosclerotic plaques in asymptomatic hyperlipidemic subjects at high cardiovascular risk. Arterioscler Thromb Vasc Biol 2003; 23: e7-10.

26. Solak B, Dikicier BS, Erdem T. Impact of elevated serum uric acid levels on systemic inflammation in patients with psoriasis. Angiology 2017; 68: 266-70. 